\title{
Does Miniaturization Actually Decrease Bleeding After Percutaneous Nephrolithotomy? A Single-Center Randomized Trial
}

\author{
Abhishek Thakur, Aditya Prakash Sharma, Sudheer Kumar Devana, Kalpesh Mahesh Parmar, \\ Ravimohan S. Mavuduru, Girdhar S. Bora, and Arup Kumar Mandal
}

\begin{abstract}
Introduction: Miniaturization of percutaneous nephrolithotomy (mPCNL) was proposed to decrease postoperative bleeding, as there is decreased parenchymal injury compared with standard percutaneous nephrolithotomy (SPCNL). Published randomized controlled trials (RCTs) evaluating bleeding after mPCNL have shown conflicting results. Hence, the evidence to say that mPCNL offers less postoperative bleeding is not robust.

Materials and Methods: Prospective RCT was done by comparing MPCNL and SPCNL for treatment of renal stones of size 1 to $3 \mathrm{~cm}$. Patients with active urinary tract infection, renal malformation, uncorrected coagulopathy, and raised serum creatinine $(>1.5 \mathrm{mg} / \mathrm{dL})$ were excluded. Blood loss was evaluated by measuring fall in hemoglobin and hematocrit on postoperative day 1. Postoperative pain was evaluated by visual analogue scale (VAS) score at 6, 24, and 48 hours.

Results: A total of 60 patients (30 in each arm) were enrolled during the study period. All the baseline preoperative variables were comparable in both the groups. The SPCNL group had a significantly higher drop in hematocrit level as compared with mPCNL $(4.6 \pm 3.1 \%$ vs $3.1 \pm 2.1 \%, p=0.02)$. The mean fall in hemoglobin was higher in SPCNL as compared with $\mathrm{mPCNL}(1.61 \pm 0.9 \mathrm{gm} \%$ vs $1.21 \pm 0.7 \mathrm{gm} \%, p=0.07)$, but it was not significant. Blood transfusion (BT) rate was comparable in both the groups (one patient in each group). The mPCNL group had significantly less VAS scores at 6 hours $(3.27 \pm 1.20 v s 4.40 \pm 1.52, p=0.002)$ and 24 hours $(2.10 \pm 0.54$ vs $2.83 \pm 1.05, p=0.001)$ after surgery. Postoperative complications and hospital stay were comparable in both the arms.

Conclusion: $\mathrm{mPCNL}$ is associated with less postoperative bleeding and pain than SPCNL. However, the small tract size does not prevent against the clinically significant bleeding requiring BT.
\end{abstract}

Keywords: MiniPCNL, miniperc, percutaneous nephrolithotomy, bleeding, randomized control trial, complications

\section{Introduction}

W ITH SIGNIFICANT ADVANCEMENT in endoscopic technology over the past three decades, minimally invasive surgery has almost replaced open surgery for the treatment of renal stones. Percutaneous nephrolithotomy (PCNL) is the treatment of choice for large renal calculi $(>20 \mathrm{~mm})$ and also the preferred treatment option for lower caliceal stones of size 10 to $20 \mathrm{~mm}$. PCNL provides stone free rates (SFRs), ranging from $76 \%$ to $98 \%$, but the most common complication of the procedure is bleeding. ${ }^{1}$ Sometimes, this bleeding may be severe enough needing a blood transfusion (BT) and angioembolization. ${ }^{2,3}$ Large diameter of the access tract, multiple access tracts, and prolonged operative time have been described as causes for increased risk of bleeding after PCNL. ${ }^{4}$ Miniaturization of PCNL (mPCNL) has come into practice in an attempt to decrease bleeding after the procedure. Various comparative studies, including randomized controlled trials (RCTs), have shown that blood loss after mPCNL is either less ${ }^{5,6}$ or comparable to standard PCNL (SPCNL). ${ }^{7}$ Wei and colleagues, in a metaanalysis comparing SPCNL with $\mathrm{mPCNL}$, showed that mPCNL resulted in less bleeding, fewer BTs, less postoperative pain, and shorter hospital stay. ${ }^{2}$ However, this metaanalysis had limitations, as most of the included studies are nonrandomized comparative studies with a high degree of

Department of Urology, Post Graduate Institute of Medical Education and Research (PGIMER), Chandigarh, India. 
heterogeneity. Hence, in the existing literature, the high level of evidence to say that mPCNL is associated with decreased blood loss is lacking. This calls for the need for better quality RCTs evaluating bleeding after SPCNL $v s$ mPCNL.

\section{Materials and Methods}

A prospective RCT (Registration number: CTRI/2018/10/ 016024) was conducted for a period of 14 months (November 2018-December 2019) comparing SPCNL with mPCNL for the treatment of renal stones with the primary objective of evaluating postoperative bleeding. Secondary objectives included postoperative pain and analgesic requirement, SFR, and complications. Patients with renal stone disease of the size of 1 to $3 \mathrm{~cm}$ planned for PCNL were evaluated for eligibility. Patients with active urinary tract infection (positive urine culture), renal malformation, uncorrected coagulopathy, morbid obesity $\left(>40 \mathrm{~kg} / \mathrm{m}^{2}\right)$, chronic kidney disease (serum creatinine $>1.5 \mathrm{mg} / \mathrm{dL}$ ), and pregnant women were excluded. All patients underwent basic investigations, which included complete hemogram, serum urea, creatinine, intravenous pyelogram (IVP), or CT urography (CTU). The stone size was defined as the maximum dimension of the stone on a preoperative IVP or CTU. In a case of multiple stones, the stone size was calculated by adding the maximum length of the individual stones. Informed consent was taken after explaining the study protocol to the patient. The sample size of 52 was calculated with an alpha error of 0.05 and a beta error of 0.2 , a power of $80 \%$ using the effect size of 0.80 for mean fall in hemoglobin in SPCNL and mPCNL. This was based on a previously published prospective comparative study on SPCNL $v s$ mPCNL in the treatment of 1 to $2 \mathrm{~cm}$ renal stones by Mishra and colleagues. ${ }^{8}$ When the maximum sample size of 60 was reached, the power of the study increased to $86 \%$. The enrolled subjects were randomized into two groups (SPCNL and mPCNL groups) by block randomization using a computer-generated random number table. After randomization, the subjects underwent either SPCNL or MPCNL by a single surgeon. The allocation was concealed in a sealed envelope and was revealed by the floor nursing staff to the operating surgeon after induction of anesthesia.

A $6 \mathrm{~F}$ ureteric catheter was initially placed up to the renal pelvis in lithotomy position, and then the patient was turned to prone position. The puncture was done under fluoroscopic guidance by using a two-part 18-gauge diamond tip PCNL puncture needle (Cook Medical, Bloomington, IN). During SPCNL, the percutaneous tract was dilated up to $24 \mathrm{~F}$ by using Alkens serial telescopic dilators and the procedure was performed by using a 22F nephroscope (Olympus, Japan). Stone fragmentation was done by using a pneumatic lithotripter probe (Swiss Lithoclast Master, Switzerland). During mPCNL the access tract was dilated up to $17.5 \mathrm{~F}$ by using a single-step metal dilator. A 12F mPCNL nephroscope of Karl Storz (Karl Storz MIP M System, Germany) was used for stone clearance. Holmium:YAG laser using a $365-\mu \mathrm{m}$ fiber was used to ablate the stones during mPCNL. A nephrostomy tube was placed in all operated cases. An $18 \mathrm{~F}$ nephrostomy tube was used for drainage in patients undergoing SPCNL, and a $12 \mathrm{~F}$ nephrostomy tube was used in the MPCNL group. Operative details such as puncture site, operative time and postoperative details such as postoperative complications and hospital stay were recorded. The SFR was assessed with kidney, ureter, and bladder radiograph (KUB) in all patients just before discharge. Complete stone clearance was defined as the absence of calculus on KUB.

Bleeding after either SPCNL or mPCNL was assessed by measuring postoperative fall in hemoglobin and hematocrit on postoperative day (POD)1. The BT, if needed before discharge, was also noted. All patients were given injection paracetamol $1 \mathrm{gm}$ intravenous 8 hourly in the postoperative period till POD1. On POD2, tablet paracetamol $650 \mathrm{mg}$ was given 8 hourly. Postoperative pain was evaluated by using visual analogue scale (VAS) score at 6, 24, and 48 hours. Patients requiring additional rescue analgesics for postoperative pain were given injection morphine $3 \mathrm{mg}$ intravenously, and these additional doses were also recorded.

Data were analyzed and reported as number and percentage or mean and standard deviation, as appropriate. All the data were analyzed by SPSS version 22 (SPSS, Inc., Chicago, IL). Fisher's exact test was used to analyze categorical data between two groups, whereas continuous data were analyzed by Student's $t$-test to compare two means. Mann-Whitney $U$-test was used for skewed data. $p$-Value $\leq 0.05$ was considered significant.

\section{Results}

A total of 90 subjects with renal stone disease were screened in the outdoor patient department and out of them, 60 subjects were enrolled to participate in the study meeting the inclusion criteria as shown in the CONSORT diagram (Fig. 1). Preoperative variables such as age, sex, body mass index, comorbidities, stone size, hemoglobin, hematocrit, serum creatinine, and stone location were comparable in both the groups (Table 1). All patients needed single percutaneous access tract for stone clearance.

The operative time $(44.17 \pm 20.55$ minutes vs $49.17 \pm$ 15.46 minutes, $p=0.29)$ and stone clearance rate $(96.6 \%$ vs $100 \%, p=0.313$ ) were comparable in both SPCNL and $\mathrm{mPCNL}$ arms, respectively. Complete clearance was achieved in all the patients of mPCNL; however, one patient in the SPCNL arm had incomplete stone clearance due to early termination of the procedure because of excessive intraoperative bleeding. The patient required BT in postoperative period as well. Fever was the most common complication occurring in both the SPCNL and mPCNL groups ( $16.6 \%$ vs $23.3 \%, p=0.13$ ). These patients had a median length of hospital stay of 3 days for SPCNL $v s 4$ days in the mPCNL group. None of the patients had features of septic shock and were managed with intravenous antibiotics only. Overall postoperative complications and hospital stay were comparable in both the arms. There was also no significant difference in postoperative serum creatinine 24 hours after surgery (Table 2).

The mean fall in hemoglobin was higher in SPCNL as compared with mPCNL $(1.61 \pm 0.9 \mathrm{gm} \%$ vs $1.21 \pm 0.7 \mathrm{gm} \%$, $p=0.07$ ), but it was not significant. Patients undergoing SPCNL had a significantly higher drop in hematocrit level as compared with mPCNL $(4.6 \pm 3.1 \%$ vs $3.1 \pm 2.1 \%$, $p=0.02$ ), as shown in Table 2. One patient each in mPCNL and SPCNL arms received $2 \mathrm{U}$ of BT for significant postoperative bleeding. None of these patients needed angioembolization for control of bleeding. Patients undergoing $\mathrm{mPCNL}$ had significantly less VAS scores at 6 and 24 hours after 


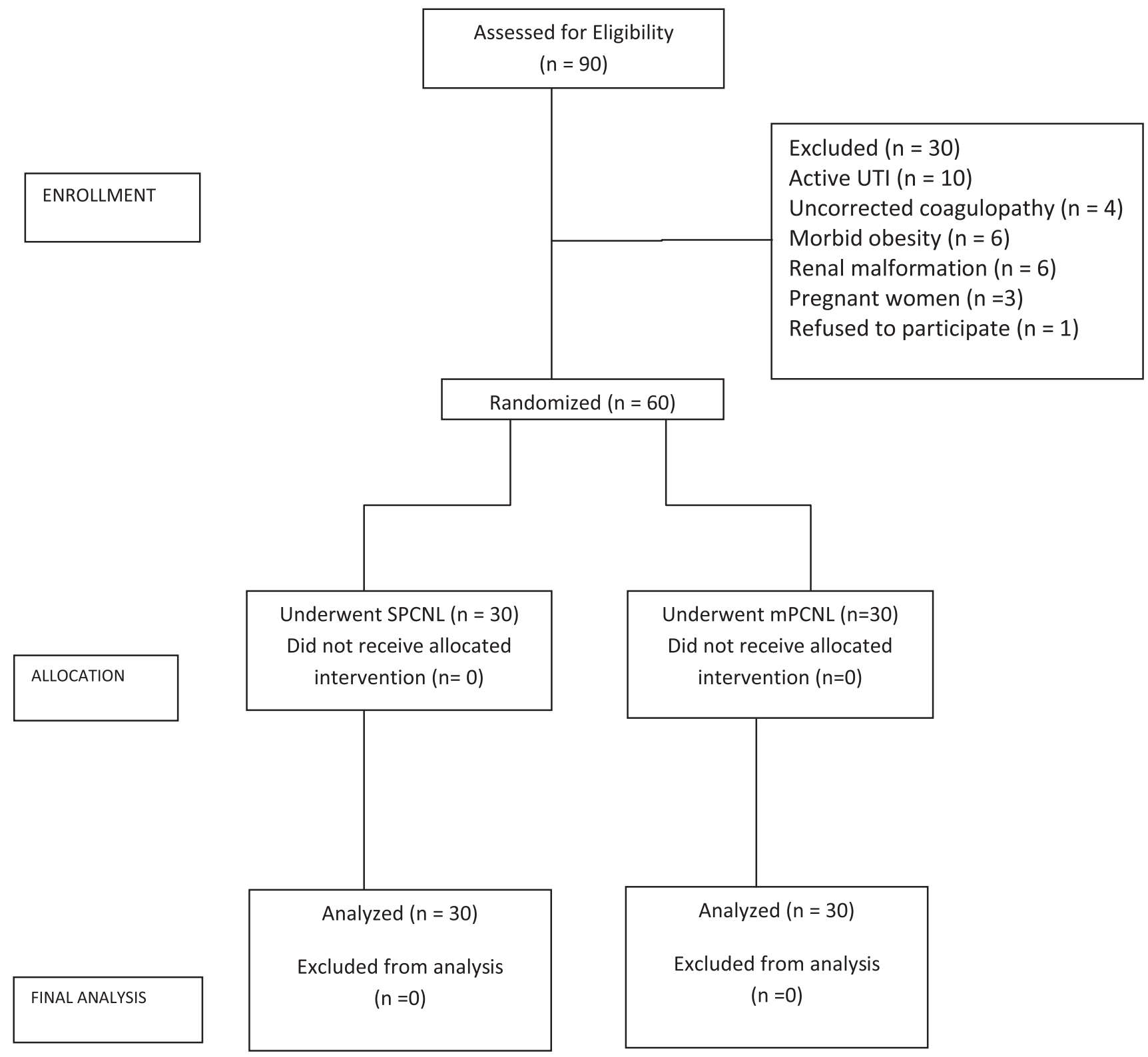

FIG. 1. Consort diagram. $\mathrm{mPCNL}=$ miniaturization of percutaneous nephrolithotomy; SPCNL $=$ standard percutaneous nephrolithotomy; UTI = urinary tract infection.

surgery compared with those undergoing SPCNL. However, the VAS scores at 48 hours of surgery were comparable between both the treatment arms (Table 2; Fig. 2). The total analgesic requirement (total dose of morphine in milligrams) for breakthrough pain in the postoperative period was higher in the SPCNL group compared with the mPCNL group, but the difference was not statistically significant $(33 \mathrm{vs} 18 \mathrm{mg}$, $p=0.22)$. Eleven patients $(36 \%)$ in the SPCNL group and one patient $(3 \%)$ in the mPCNL group had more than moderate pain after the surgery.

\section{Discussion}

mPCNL has been proposed to be associated with less bleeding, as it causes less renal parenchymal injury. However, the evidence for this in the existing literature is con- flicting. Cheng and colleagues, in an RCT comparing mPCNL $(n=72)$ with SPCNL $(n=115)$, had shown a significantly lower fall in hemoglobin levels in the mPCNL group (0.53 vs $0.97 \mathrm{gm} / \mathrm{dL}, p<0.05){ }^{5}$ Similarly, an RCT by Song and colleagues also found that the mPCNL group had a significantly less amount of intraoperative bleeding as compared with the SPCNL group $(90.99 \pm 33.1 \mathrm{~mL}$ vs $110.9 \pm$ $35.2 \mathrm{~mL}, p=0.01){ }^{6}$ However, in their study, there was a significant difference in the number of access tracts used for PCNL between both the groups $(p<0.0018)$. Contrary to this, Zhong and colleagues had shown that in patients with staghorn stones, a hemoglobin drop after mPCNL $v s$ SPCNL (3.2 vs $3.5 \mathrm{~g} / \mathrm{dL}, p=0.09$ ) was not significantly different. ${ }^{7}$ In another multicenter RCT by Zeng, no significant difference was observed in the hematocrit drop between mPCNL and SPCNL groups for 2 to $4 \mathrm{~cm}$ size kidney stones. ${ }^{9}$ Hence, the 
Table 1. Demographic Data of the Study Participants

\begin{tabular}{|c|c|c|c|}
\hline & $\begin{array}{l}S P C N L \\
(\mathrm{~N}=30)\end{array}$ & $\begin{array}{l}m P C N L \\
(\mathrm{~N}=30)\end{array}$ & $\mathrm{p}$ \\
\hline Age (years), mean \pm SD & $32.4 \pm 12.6$ & $34.5 \pm 16.32$ & 0.58 \\
\hline Sex & & & \\
\hline $\begin{array}{l}\text { Males } \\
\text { Females }\end{array}$ & $\begin{array}{l}17 \\
13\end{array}$ & $\begin{array}{c}21 \\
9\end{array}$ & $\begin{array}{l}0.76 \\
0.32\end{array}$ \\
\hline $\begin{array}{l}\mathrm{BMI}\left(\mathrm{kg} / \mathrm{m}^{2}\right) \\
\text { mean } \pm \mathrm{SD}\end{array}$ & $25 \pm 5.16$ & $26.32 \pm 5.10$ & 0.33 \\
\hline $\begin{array}{l}\text { Presence of } \\
\quad \text { comorbidities, } n(\%)\end{array}$ & $3(1)$ & $3(1)$ & \\
\hline $\begin{array}{l}\text { Preoperative } \\
\text { hemoglobin }(\mathrm{gm}, \%) \text {, } \\
\text { mean } \pm \text { SD }\end{array}$ & $12.84 \pm 2.00$ & $13.07 \pm 1.98$ & 0.65 \\
\hline $\begin{array}{l}\text { Preoperative hematocrit, } \\
\text { mean } \pm S D\end{array}$ & $38.66 \pm 6.68$ & $38.61 \pm 5.40$ & 0.97 \\
\hline $\begin{array}{l}\text { Stone size }(\mathrm{cm}) \\
\text { mean } \pm \mathrm{SD}\end{array}$ & $1.94 \pm 0.53$ & $1.79 \pm 0.50$ & 0.226 \\
\hline $\begin{array}{l}\text { Serum creatinine } \\
\quad(\mathrm{mg} / \mathrm{dL}) \text {, mean } \pm \mathrm{SD}\end{array}$ & $0.87 \pm 0.40$ & $0.95 \pm 0.49$ & 0.49 \\
\hline \multicolumn{4}{|l|}{ Stone location, $n(\%)$} \\
\hline Superior calix & $1(3.3)$ & 0 & 0.15 \\
\hline Middle calix & 0 & $4(13.3)$ & \\
\hline Inferior calix & $5(16.7)$ & $4(13.3)$ & \\
\hline Renal pelvis & $24(80)$ & $22(73.3)$ & \\
\hline
\end{tabular}

$\mathrm{BMI}=$ body mass index $; \mathrm{mPCNL}=$ miniaturization of percutaneous nephrolithotomy; $\mathrm{SD}=$ standard deviation; $\mathrm{SPCNL}=$ standard percutaneous nephrolithotomy.

evidence regarding whether $\mathrm{mPCNL}$ has less bleeding than SPCNL is at best controversial as of now.

We conducted this RCT comparing SPCNL with mPCNL for the treatment of renal stone disease, evaluating postoperative bleeding and pain by using strict selection criteria. As generally $\mathrm{mPCNL}$ is recommended for renal stones with less stone burden, we have specifically enrolled subjects with a maximum stone size of 1 to $3 \mathrm{~cm}$ and excluded patients with larger stones/staghorn stones. Subjects with serum creatinine $>1.5 \mathrm{mg} / \mathrm{dL}$ and those with coagulopathy were also excluded. All patients in both the treatment arms in our study had a comparable stone size and needed only a single percutaneous tract for stone clearance. It is worth noting that the operative time that has an effect on bleeding was also comparable in both the arms. We found that postoperative bleeding as measured by a fall in hematocrit $(3.1 \pm 2.1$ vs $4.6 \pm 3.1, p=0.02)$ and hemoglobin $(1.21 \pm 0.7 \mathrm{gm} \%$ vs $1.61 \pm 0.91 \mathrm{gm} \%, p=0.07)$ was less in mPCNL compared with SPCNL. It is prudent to mention here that the fall in the hematocrit level after PCNL is a better marker to assess postoperative bleeding than the fall in hemoglobin level, as the latter can be affected by the amount of intravenous fluids being transfused in the perioperative period. Hence, based solely on the hematocrit level, our study definitely shows that mPCNL is associated with significantly less bleeding compared with SPCNL. However, these changes in hemoglobin and hematocrit are not clinically meaningful, as they did not warrant any intervention such as BT, which is discussed further.

The clinical significance of blood loss is reflected by the need for BT. The BT rates were comparable in MPCNL and SPCNL arms in our study. This suggests that severe bleeding (needing BT) is comparable in both the treatment arms. One

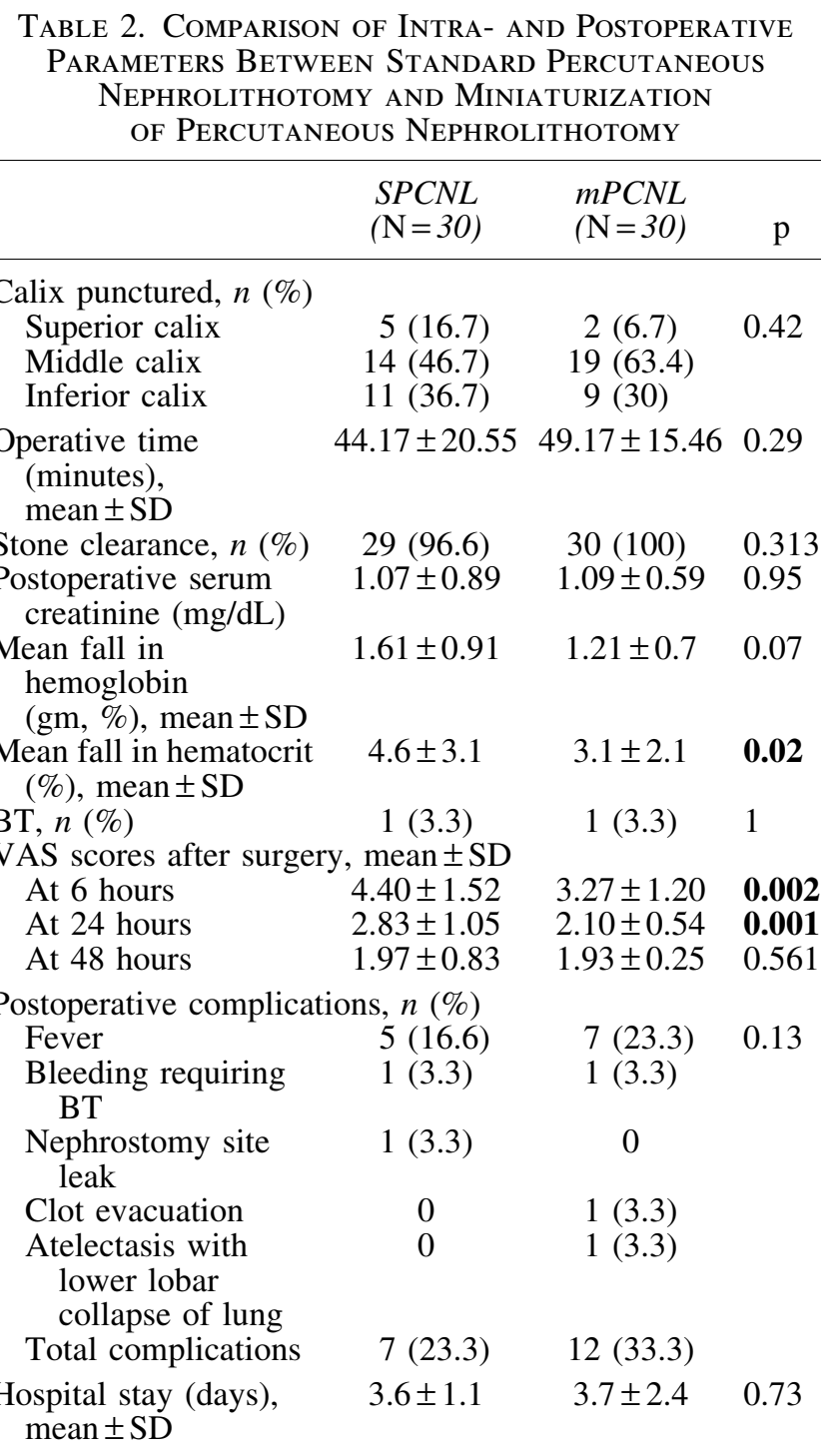

$\mathrm{BT}=$ blood transfusion $; \mathrm{VAS}=$ visual analogue scale.

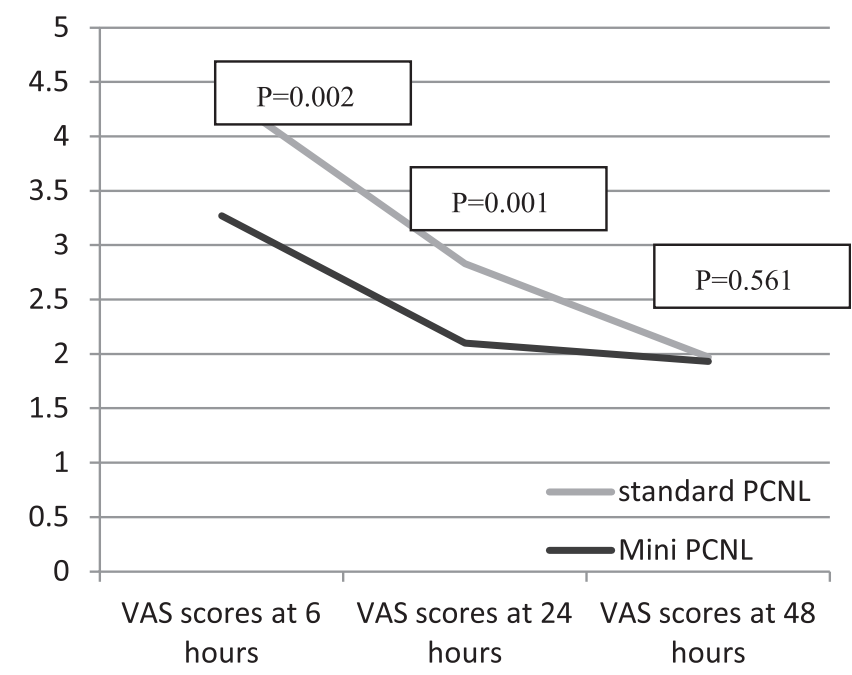

FIG. 2. Postoperative pain assessment with VAS scores. VAS $=$ visual analogue scale. 
patient in the mPCNL arm had sudden onset of bleeding on POD1, with a significant fall in hemoglobin needing clot evacuation and $2 \mathrm{U}$ of BT. Similarly, one patient in SPCNL had persistent bleeding during the surgery, with hypotension in the immediate postoperative period that was conservatively managed with $2 \mathrm{U}$ of BT. The same patient had a residual calculus in the non-punctured calix. None of these patients needed angioembolization for control of bleeding. Increased bleeding in these two cases could be attributed to injury to a blood vessel during puncture and dilatation of access tract. This suggests that along with the size of the percutaneous access tract, the initial puncture is very important in the prevention of severe bleeding after PCNL. Hence, it is clear that patients undergoing mPCNL are not immune to severe postoperative bleeding. This is further corroborated from the fact that even renal biopsies done with an 18-gauge biopsy needle in comparison to the mPCNL access tract $(12 \mathrm{~F}-20 \mathrm{~F})$ can also cause severe post-procedure bleeding requiring transfusion, angioembolization, and rarely nephrectomy. ${ }^{10}$ Ideal caliceal puncture through the papilla of the calix in line with the axis of the infundibulum is a prerequisite for preventing significant bleeding even in mPCNL cases. It may further be stated that type of dilatation, single step $v s$ serial dilatation could also affect bleeding after PCNL. In a meta-analysis comparing one-step dilatation with serial dilatation, Peng and colleagues found a significant difference in hemoglobin fall. ${ }^{11}$ They reported a higher fall in the serial dilatation group ( $\mathrm{MD}=0.23 ; 95 \%$ confidence interval -0.39 to $-0.07 ; p=0.004$ ). However, similar to our study, the $\mathrm{BT}$ rate was comparable in both the groups.

mPCNL has also been proposed to be associated with less postoperative pain and analgesic requirement compared with SPCNL. ${ }^{5,12}$ Smaller incision, smaller percutaneous tract, and increased chance of tubeless procedure during mPCNL are described as the probable reasons for less pain after mPCNL ${ }^{13}$ According to Pietrow and colleagues, placement of a smaller-sized nephrostomy tube during mPCNL has also been shown as a probable reason for reduced postoperative pain. ${ }^{14}$ Contrary to the studies just cited, RCT by Cheng and colleagues had shown no significant difference in VAS scores on POD1 in mPCNL as compared with SPCNL $(4.3 \pm 2.1 v \mathrm{~s}$ $4.3 \pm 2.5, p>0.05) .{ }^{5}$ Similarly, there are other nonrandomized studies that have also shown no significant difference in pain after mPCNL when compared with SPCNL. ${ }^{12}$ However, in our study, we noticed that VAS was significantly less after the mPCNL group compared with the SPCNL group at 6 and 24 hours after surgery. This was also reflected in the lesser postoperative analgesic requirement in the mPCNL arm compared with the SPCNL arm. In spite of placement of nephrostomies (18F in SPCNL vs $12 \mathrm{~F}$ in mPCNL) in all our study participants, we observed significantly less postoperative pain in patients undergoing $\mathrm{mPCNL}$. This suggests that a smaller access tract and smaller nephrostomy tube are associated with lesser pain after mPCNL in the immediate postoperative period. However, at 48 hours after surgery, the postoperative pain was comparable in both mPCNL and SPCNL. The probable reason for this could be that in the majority of our patients the nephrostomy tube is removed 24 hours after the surgery and the nephrostomy tube is one of the very important determinants of VAS scores in PCNL patients.

Derangement in renal function was documented in the early postoperative period after PCNL by Mukherjee and colleagues. ${ }^{15}$ In their study, Handa and colleagues showed a significant rise in serum creatinine 24 hours after PCNL. ${ }^{16}$ We also found an increase in serum creatinine at 24 hours postoperatively; however, the postoperative serum creatinine was not significantly different in either of the groups. Metaanalysis by Zhu and colleagues has shown that the SFR was not significantly different in mPCNL and SPCNL. ${ }^{2}$ Similarly, in our study, also SFR was comparable in both the treatment groups $(100 \%$ vs $96.6 \%, p=0.313)$. mPCNL had higher complications than SPCNL, although not reaching statistical significance $(36.4 \%$ vs $19.9 \%, p=0.13)$, similar to the previously published literature. ${ }^{5-7}$ The most common complication was fever in both groups. Decreased hospital stay in mPCNL as reported by Giusti and colleagues ${ }^{12}$ and Zeng ${ }^{9}$ is due to lesser need for nephrostomy placement during $\mathrm{mPCNL}$, as it is associated with less bleeding. Since there is no nephrostomy tube, patients undergoing mPCNL get discharged early from the hospital. However, in our study, hospital stay was comparable between the two groups (3.7 \pm 2.4 days $v s 3.6 \pm 1.6$ days, $p=0.7$ ) as the nephrostomy tube was placed in all operated patients. The hospital stay is also affected by hospital and insurance policies that are prevalent at a particular center. Since we are at a tertiary care government hospital, all patients were discharged only after nephrostomy removal.

Our study is unique compared with the previously published RCTs comparing mPCNL and SPCNL in a few aspects. Homogenous study participants with a renal stone size of 1 to $3 \mathrm{~cm}$ only were included. We also state that the small size of stones as depicted in this study would also affect the SFR and operative time. For stones $<2 \mathrm{~cm}$, shockwave lithotripsy and retrograde intrarenal surgery (RIRS) also remain a viable option, though they were not a part of this study. Even in our study, out of 30 patients, we excluded four patients with uncorrected coagulopathy, six patients with morbid obesity, and six patients with renal malformation who underwent RIRS. Factors affecting postoperative bleeding after PCNL, such as the number of percutaneous access tracts and operative time, were comparable in both the treatment arms. Since presence or absence of the nephrostomy tube can affect postoperative pain scores, we deliberately placed a nephrostomy tube in both the study groups so as not to bias our results. However, a limitation of our study is that we did not evaluate the intravenous fluid transfusion status of operated patients, which might affect the postoperative hemoglobin levels.

\section{Conclusion}

mPCNL is associated with less postoperative bleeding and pain than SPCNL. However, the small tract size does not prevent against the clinically significant bleeding requiring BT.

\section{Author Disclosure Statement}

No competing financial interests exist.

\section{Funding Information}

No funding information was received.

\section{References}

1. Michel MS, Trojan L, Rassweiler JJ. Complications in percutaneous nephrolithotomy. Eur Urol 2007;51:899-906. 
2. Zhu W, Liu Y, Liu L, Lei M, Yuan J, Wan SP, Zeng G. Minimally invasive versus standard percutaneous nephrolithotomy: A meta-analysis. Urolithiasis 2015;43:563-570.

3. El Tayeb MM, Knoedler JJ, Krambeck AE, Paonessa JE, Mellon MJ, Lingeman JE. Vascular complications after percutaneous nephrolithotomy: 10 Years of experience. Urology 2015;85:777-781.

4. Yamaguchi A, Skolarikos A, Buchholz NP, Chomon GB, Grasso M, Saba P, Nakada S, de la Rosette, on behalf of the Clinical Research Office of the Endourological Society Percutaneous Nephrolithotomy Study Group. Operating times and bleeding complications in percutaneous nephrolithotomy: A comparison of tract dilatation methods in 5537 patients in the Clinical Research Office of the Endourological Society Percutaneous Nephrolithotomy Global Study. J Endourol 2011;25:933-939.

5. Cheng F, Yu W, Zhang X, Yang S, Xia Y, Ruan Y. Minimally invasive tract in percutaneous nephrolithotomy for renal stones. J Endourol 2010;24:1579-1582.

6. Song L, Chen Z, Liu T, et al. The application of a patented system to minimally invasive percutaneous nephrolithotomy. J Endourol 2011;25:1281-1286.

7. Zhong W, Zeng G, Wu W, Chen W, Wu K. Minimally invasive percutaneous nephrolithotomy with multiple mini tracts in a single session in treating staghorn calculi. Urol Res 2011;39:117-122.

8. Mishra S, Sharma R, Garg C, Kurien A, Sabnis R, Desai M. Prospective comparative study of miniperc and standard PNL for treatment of 1 to $2 \mathrm{~cm}$ size renal stone. BJU Int 2011;108:896-900.

9. Zeng G. Mini-PCNL versus standard-PCNL for the management of 20-40 mm size kidney stones: The initial result of a multi-center randomized controlled trial. Eur Urol Suppl 2018;17:e1224.

10. Manno C, Strippoli GF, Arnesano L, Bonifati C, Campobasso N, Gesualdo L, Schena FP. Predictors of bleeding complications in percutaneous ultrasound-guided renal biopsy. Kidney Int 2004;66:1570-1577.

11. Peng PX, Lai SC, Ding ZS, He YH, Zhou LH, Wang XM, Zhang G. One-shot dilation versus serial dilation technique for access in percutaneous nephrolithotomy: A systematic review and meta-analysis. BMJ Open 2019;9: e025871.

12. Giusti G, Piccinelli A, Taverna G, et al. Miniperc? No, thank you! EurUrol 2007;51:810-814.
13. Knoll T, Wezel F, Michel MS, Honeck P, Wendt-Nordahl G. Do patients benefit from miniaturized tubeless percutaneous nephrolithotomy? A comparative prospective study. J Endourol 2010;24:1075-1079.

14. Pietrow PK, Auge BK, Lallas CD, Santa-Cruz RW, Newman GE, Albala DM, Preminger GM. Pain after percutaneous nephrolithotomy: Impact of nephrostomy tube size. J Endourol 2003;17:411-414.

15. Mukherjee S, Sinha RK, Jindal T, Sharma PK, Mandal SN, Karmakar D. Short-term alteration of renal function and electrolytes after percutaneous nephrolithotomy. Urol J 2019;16:530-535.

16. Handa RK, Matlaga BR, Connors BA, et al. Acute effects of percutaneous tract dilation on renal function and structure. J Endourol 2006;20:1030-1040.

Address correspondence to: Sudheer Kumar Devana Department of Urology

Post Graduate Institute of Medical Education and Research (PGIMER) Chandigarh 160012 India

E-mail: drsudheer1983@gmail.com

$\begin{aligned} & \text { Abbreviations Used } \\ \mathrm{BMI} & =\text { body mass index } \\ \mathrm{BT} & =\text { blood transfusion } \\ \mathrm{CT} & =\text { computed tomography } \\ \mathrm{CTU} & =\mathrm{CT} \text { urography } \\ \mathrm{IVP} & =\text { intravenous pyelogram } \\ \mathrm{KUB} & =\text { kidney, ureter, and bladder radiograph } \\ \mathrm{mPCNL} & =\text { miniaturization of PCNL } \\ \mathrm{PCNL} & =\text { percutaneous nephrolithotomy } \\ \mathrm{POD} & =\text { postoperative day } \\ \mathrm{RCT} & =\text { randomized controlled trial } \\ \mathrm{RIRS} & =\text { retrograde intrarenal surgery } \\ \mathrm{SD} & =\text { standard deviation } \\ \mathrm{SFR} & =\text { stone-free rate } \\ \mathrm{SPCNL} & =\text { standard PCNL } \\ \mathrm{UTI} & =\text { urinary tract infection } \\ \mathrm{VAS} & =\text { visual analogue scale }\end{aligned}$

